\title{
Mitigating the bycatch of giant cuttlefish Sepia apama and blue swimmer crabs Portunus armatus in an Australian penaeid-trawl fishery
}

\author{
Steven J. Kennelly ${ }^{1, *}$, Matt K. Broadhurst ${ }^{2}$ \\ ${ }^{1}$ IC Independent Consulting, 15/1-7 Arthur Ave, Cronulla, NSW 2230, Australia \\ ${ }^{2}$ NSW Department of Primary Industries, PO Box 4321, Coffs Harbour, NSW 2450, Australia
}

\begin{abstract}
Trawlers in Spencer Gulf, Australia, target western king prawns Melicertus latisulcatus, with nearly all other species discarded; two of the latter have evoked significant, although quite different, concerns. The first is the giant cuttlefish Sepia apama, which has undergone a drastic decline in numbers in Spencer Gulf in recent years, and is listed as 'Near Threatened' by the International Union for the Conservation of Nature. S. apama are susceptible to trawling during their annual spawning migration through Spencer Gulf. The other bycatch problem involves large quantities of blue swimmer crabs Portunus armatus, which currently are separated inside the trawl using a large-meshed liner. Due to the additional handling required and the physical damage caused by the crabs' exoskeletons on the soft-bodied M. latisulcatus and Sepia spp., $P$. armatus would ideally be able to escape of their own accord during towing. This study examined the utility of mechanical-separating bycatch reduction devices (BRDs) for reducing unwanted bycatches of Sepia spp. and P. armatus. We compared (against conventional codends) the smallest and largest practical sizes of Nordmøre-grids (the latter to maximise sorting area), with correspondingly large and low grid angles. The large Nordmøre-grid significantly reduced Sepia spp., $P$. armatus and total bycatch (by 33-50\%), but had no effect on catches of M. latisulcatus. Whilst additional research is required, a modified Nordmøre-grid should help to resolve the bycatch of $P$. armatus and $S$. apama in this fishery, with minimal commercial impacts.
\end{abstract}

KEY WORDS: Bycatch • Discards · Bycatch reduction devices · Giant cuttlefish • Crabs · Prawn trawl

\section{INTRODUCTION}

Demersal trawls are the main commercial fishing gear used to target penaeids; however, this method is generally regarded as being among the world's most poorly selective (Alverson et al. 1994, Kelleher 2005). Issues concerning trawl bycatch have attracted significant attention, controversy and priority for decades. A major concern among many fisheries has been the bycatch of endangered species such as sea turtles. More regionally, the mortality of fisheryspecific, charismatic and/or other threatened species

\footnotetext{
${ }^{*}$ Corresponding author: steve.kennelly@icic.net.au
}

is often a challenge for local resource management (Hall 1996).

Considerable ongoing efforts have been directed towards improving the sustainability of penaeidtrawl fisheries, especially in developed countries. In Australia, one such example is the South Australian Spencer Gulf prawn fishery, which has Marine Stewardship Council certification and sophisticated selfmanagement involving real-time spatial and temporal effort regulation (including only 50-55 nights of fishing $\mathrm{yr}^{-1}$ ). Operators are only permitted to land western king prawns Melicertus latisulcatus, slipper

() The authors 2014. Open Access under Creative Commons by Attribution Licence. Use, distribution and reproduction are unrestricted. Authors and original publication must be credited. 
lobsters Ibacus spp. and southern calamari Sepioteuthis australis. The remaining discarded bycatch comprises a diverse assemblage of species, 2 of which, i.e. (1) giant cuttlefish Sepia apama and (2) blue swimmer crabs Portunus armatus, have evoked particular concerns for quite different reasons.

The $S$. apama issue in this fishery recently arose due to a marked decline in the local population from a peak of $\sim 183000$ spawning animals in 1999 to an estimated 18530 in 2012 (Steer et al. 2013). This decline led to significant public consternation, media attention and the species being listed by the International Union for Conservation of Nature as 'Near Threatened' (Barratt \& Allcock 2012). While definitive causes of the population decline are unknown, the remaining populations of $S$. apama $(30-250 \mathrm{~mm}$ mantle length [ML]) migrate through very productive trawling grounds in the Spencer Gulf from March to May of each year and are caught by trawlers. This interaction led to the current project to examine ways of reducing the bycatch of $S$. apama. Complicating this issue is that 2 other species of unthreatened cuttlefish, $S$. novaehollandae and $S$. braggi, are also trawled and discarded, but are morphologically similar to $S$. apama and can only be distinguished by lethal internal examination (Dixon et al. 2014).

The other, longer-standing bycatch problem involves large quantities of $P$. armatus $(30-150 \mathrm{~mm}$ carapace width [CW]) which are targeted by commercial and recreational trap fishers, but must be discarded by the trawl fishery. Currently, trawled $P$. armatus are separated inside trawls using a largemeshed liner (termed a 'crab bag') terminating anterior to the end of the codend. Most M. latisulcatus, $S$. australis and Ibacus spp. pass through the crab bag into the codend, whilst larger $P$. armatus are retained in it. All $P$. armatus caught in the crab bag are then landed on the vessel, quickly sorted and discarded, and, because any associated mortality is expected to be low, the crab bag is considered an appropriate strategy for resolving this particular bycatch issue. However, due to (1) the additional handling required and (2) the physical damage caused by the crabs' exoskeletons on the soft-bodied M. latisulcatus, S. australis and Sepia spp., ideally $P$. armatus would be able to escape of their own accord from the trawls during towing.

During the past $30 \mathrm{yr}$, numerous bycatch reduction devices (BRDs) have been developed for penaeidtrawl fisheries throughout the world to resolve various selectivity issues, including those concerning threatened species (for reviews see Broadhurst 2000, Kennelly 2007). Of particular success have been mechanical-separating BRDs that mostly partition the catch according to size differences (e.g. the 'Nordmøre-grid' described by Isaksen et al. 1992). These BRDs comprise a rigid grid with appropriate bar spacings, typically installed at $\sim 45^{\circ}$ in the codend and terminating in an escape exit. The utility of such designs is largely determined by the relative size/ morphology (and in some cases behaviour) of the penaeids and bycatch species, which, in many cases, often overlap-including for those species in the Spencer Gulf fishery.

Over the last $20 \mathrm{yr}$, several mechanical-separating BRDs have been tested in Spencer Gulf (mainly to exclude $P$. armatus), but none have been implemented. Early work involved trials of various grids and American designs (used to exclude sea turtles in other fisheries) including a 'super-shooter', a 'Seymour grid' and a version of the Nordmøre-grid (McShane 1997). More recent work examined modifications of such designs (Dixon et al. 2014). Whilst some of these modifications significantly reduced bycatches, they did so inconsistently, and unacceptable losses of $M$. latisulcatus precluded their implementation.

The purpose of the current study was to identify the maximum and minimum physical dimensions of a mechanical-separating BRD which will reduce the bycatch of Sepia spp. and P. armatus in the Spencer Gulf fishery, whilst still maintaining conventional catches of M. latisulcatus. Note that because S. apa$m a, S$. novaehollandae and $S$. braggi are morphologically similar, any such BRD will need to exclude all 3 species. Our approach involved comparing what was considered to be the smallest and largest practical grid sizes (the latter to maximise sorting area) and with correspondingly large and low grid angles (to maintain identical codend geometry). The logic underpinning this approach was that, if a large, lowangled grid could not facilitate the escape of Sepia spp. and $P$. armatus whilst maintaining catches of M. latisulcatus, then mechanical-separating BRDs may not be appropriate for resolving these issues in this fishery.

\section{MATERIALS AND METHODS}

This study was carried out in April 2014 (during the Sepia spp. migration) in Spencer Gulf, South Australia $\left(31.42^{\circ} \mathrm{S}, 136.75^{\circ} \mathrm{E}\right)$, using a double-rigged trawler $(22 \mathrm{~m}$ and $330 \mathrm{~kW}$ ) fishing in $10-30 \mathrm{~m}$ across sandy substrata. The experiment was purposely done in areas where, based on the skipper's experi- 
ence, typical catches for this time of year (i.e. during the $S$. apama migration) of Melicertus latisulcatus, Sepia spp. and Portunus armatus were likely to be encountered.

The trawler had 2 identical 'Gundry' trawls with $14.63 \mathrm{~m}$ headline lengths; each was spread by flatrectangular otter boards $(0.9 \times 2.7 \mathrm{~m})$ and towed at $\sim 1.8 \mathrm{~m} \mathrm{~s}^{-1}$. Both posterior trawl bodies had zippers attached (Buraschi S146R, $2.0 \mathrm{~m}$ long) to facilitate changing extension/codend sections. Two extensions and 3 codends were constructed (Fig. 1A). The extensions were made of identical $41 \mathrm{~mm}$ mesh (3.0 mm diameter braided black twine), measured with 150 meshes in a transversal direction (T) and 95 and 110 meshes in a normal direction $(\mathrm{N})$, respectively, and had guiding panels following the specifications provided by Broadhurst et al. (1997) (Fig. 1A). Two Nordmøre-grids were inserted into the posterior
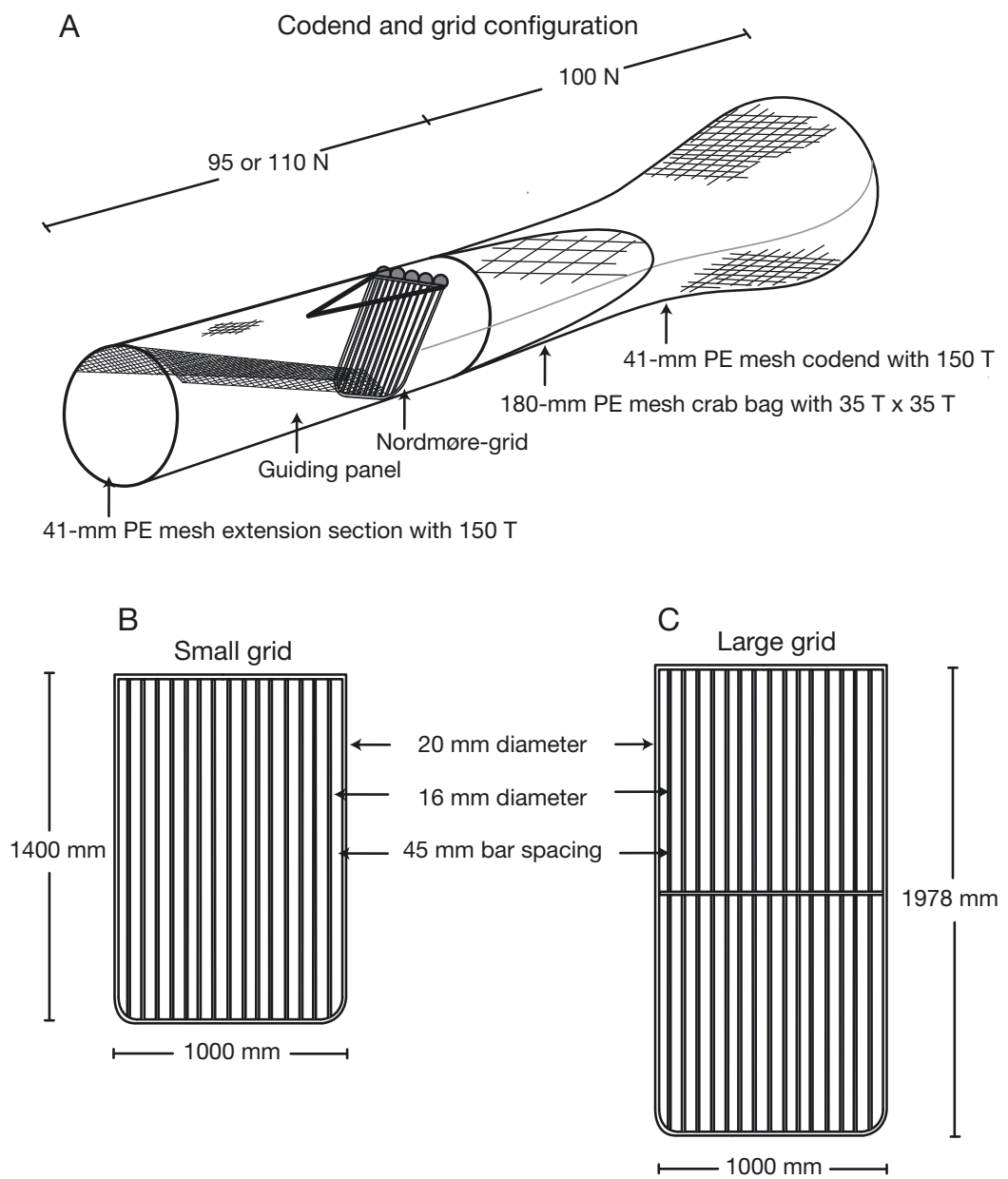

Fig. 1. Schematic representations of (A) a codend and extension (with crab bag) with a Nordmøre-grid installed, and the (B) small and (C) large Nordmøre-grids. Number of meshes in a normal direction $(\mathrm{N})$ and a transversal direction (T) are shown; PE: polyethylene extension sections (at the base of the guiding panels), and triangular escape exits were cut (Fig. 1A). Both Nordmøre-grids were made of solid aluminium rod (20 and $16 \mathrm{~mm}$ frame and bars, respectively), with $45 \mathrm{~mm}$ bar spaces, and measured $1.0 \mathrm{~m}$ in width (Fig. 1B,C). The first Nordmøre-grid (termed 'small') was broadly based on those tested in other fisheries, with an aspect ratio of $\sim 1: 1.4(1.40 \mathrm{~m}$ long) and was located in the extension at an angle of $\sim 45^{\circ}$ (for installation instructions see Broadhurst et al. 1996, 1997, Silva et al. 2011; Fig. 1B)). The second grid (termed 'large') measured $1.98 \mathrm{~m}$ long and was positioned at $\sim 30^{\circ}$ (Fig. 1C). Both grids were installed so that they had identical fishing heights (i.e. had the same extension/codend diameter) and were rigged with sufficient $200 \mathrm{~mm}$ diameter polyurethane floats (behind the upper frame) to achieve neutral buoyancy.

The codends were conventional designs, made of identical $41 \mathrm{~mm}$ mesh $(2.2 \mathrm{~mm}$ diameter braided green twine), and measured $150 \mathrm{~T} \times 100 \mathrm{~N}$. Each codend had a crab bag, made of $180 \mathrm{~mm}$ braided mesh, measuring $35 \mathrm{~T} \times 35 \mathrm{~N}$ inserted into the anterior section (see Fig. 1A). Two of the codends were attached directly posterior to the Nordmøre-grids, while the third was used as a control.

Between 19:00 and $05: 30 \mathrm{~h}$ on 3 fishing nights, the small and large Nordmøre-grids were tested against the control during simultaneous, paired 30 min deployments ( 8 and 7 , respectively), alternating on each side of the vessel (NB the fishery is exclusively a night-time fishery, and $30 \mathrm{~min}$ is the standard tow time used by the fleet). At the end of each deployment, data were collected on: the total weights of $M$. latisulcatus and various industry size grades that included under 8 (U8), under 10 (U10), 11-15, 16-20 and 21-30 individuals per pound ( 1 pound $=454 \mathrm{~g}$ ), and any damaged or post-moult individuals (termed 'soft'); the weight of $S$. australis; the weights and numbers of Sepia spp. and P. armatus (representative sub-sampling was required to estimate the numbers of the latter); and the weight of the remaining (termed 'mixed') and total bycatch. 
Data were log-transformed so that the predicted effects would be multiplicative and analysed in linear mixed models (LMMs) that included 'codend' as a fixed effect, while 'sides', 'nights' and the interaction between 'nights' and 'deployments' were included as random terms. The models were fitted using the ASReml package in the $R$ statistical language, with the significance of codend determined using a Wald F-statistic. Any significant effects were subsequently explored using the Benjamini-Hochberg-Yekutieli procedure to control the false discovery rate (FDR) for multiple pair-wise comparisons.

\section{RESULTS}

A total of 1801, 59 and $2026 \mathrm{~kg}$ of Melicertus latisulcatus, retained Sepioteuthis australis and total bycatch, respectively, were caught during the 15 paired deployments. Sepia spp. ( 30-250 mm ML) and Portunus armatus ( 30-150 mm CW) comprised 5.4 and $64.7 \%$ of the total bycatch and 2.8 and $33.4 \%$ of the total biomass caught, respectively. The latter compares with the only other estimates of bycatch in this fishery made during a fishery-independent survey (Currie et al. 2009) which revealed biomass ratios of 0.73 and $17.2 \%$ for Sepia spp. and P. armatus, respectively. Although not quantified, the mixed subset of bycatch $(32.8 \%$; and excluding Sepia spp. and $P$. armatus) had several small teleosts (most $<20 \mathrm{~cm}$ total length [TL]), including sand trevally Pseudocaranx wrighti, red mullet $U p$ eneichthys porosus, school whiting Sillago bassensis, southern sand flathead Platycephalus bassensis, Degen's leather jacket Thamnaconus degeni and small-toothed flounder Pseudorhombus jenynsii.

The LMMs detected significant differences among codends for the weights of total M. latisulcatus and the 11-15 and 21-30 per pound subcategories, the numbers and weights of Sepia spp. and P. armatus and the weight of total bycatch ( $\mathrm{p}<0.05$; Table 1$)$. For M. latisulcatus, the significant codend effect was largely explained by a slight reduction in predicted mean weights by the small grid $(7.8 \%)$ compared to the large grid and control (which had identical catches; FDR, $\mathrm{p}<0.05$; Table 1). The remaining differences in catches reflected significant and comparable reductions by both Nordmøre-grids compared to the control, including the numbers and weights of Sepia spp. (by 50.4 and $59.7 \%$ respectively, for the small grid, and 33.5 and $36.6 \%$, respectively, for the large grid), $P$. armatus (by 40.2 and $47.5 \%$, respectively, for the small grid, and 33.9 and $50.1 \%$, respectively, for the large grid) and total bycatch (by $40.9 \%$ for the small grid and $38.1 \%$ for the large grid) (FDR, p < 0.001; Table 1). The only variables not affected by codend configuration were $S$. australis and mixed bycatch (i.e. teleosts less than $\sim 20 \mathrm{~cm}$ TL) (LMM, p > 0.05; Table 1).

In addition to the above quantitative data, we also gathered information during the experiment concerning the operation of the BRDs. Despite the size of the grids (especially the large one), there were few problems encountered during deployment, and ondeck handling was relatively straightforward. However, during haulback, we noticed some clogging of the guiding panel close to the small grid, but less so for the large grid. This clogging of all species was mainly caused by $P$. armatus clinging or being entangled, and was included in the catch sampled for each replicate.

Table 1. Wald $F$-values from linear mixed models assessing the significance of codend (S: small grid; L: large grid; C: control) on key variables, predicted back-transformed (from the log scale) means (kilograms and numbers per 30 min deployment) and falsediscovery-rate (FDR)-adjusted paired comparisons of significant differences. Melicertus latisulcatus categories are industry counts per pound (454 g). -: not relevant; U8 and U10: under 8 or 10 individuals per pound, respectively; 'soft': damaged or postmoult individuals; ${ }^{*} \mathrm{p}<0.05 ;{ }^{* *} \mathrm{p}<0.01 i^{* * *} \mathrm{p}<0.001$

\begin{tabular}{|c|c|c|c|c|c|}
\hline \multirow{2}{*}{ Variable } & \multirow[b]{2}{*}{$\begin{array}{l}\text { Wald } \\
\text { F }\end{array}$} & \multirow{2}{*}{$\begin{array}{c}\text { Small } \\
\text { grid }\end{array}$} & \multicolumn{2}{|c|}{ Predicted means } & \multirow[b]{2}{*}{ FDR } \\
\hline & & & $\begin{array}{l}\text { Large } \\
\text { grid }\end{array}$ & Control & \\
\hline \multicolumn{6}{|l|}{ Wt. of Melicertus latisulcatus } \\
\hline U8 & 0.05 & 3.10 & 2.9 & 3.01 & - \\
\hline U10 & 0.49 & 3.60 & 3.46 & 3.47 & - \\
\hline $11-15$ & $4.49^{*}$ & 20.29 & 21.97 & 26.73 & None detected \\
\hline $16-20$ & 2.43 & 48.58 & 53.1 & 51.86 & - \\
\hline $21-30$ & $5.00^{*}$ & 10.23 & 12.13 & 11.81 & $\mathrm{~S}<\mathrm{L}=\mathrm{C}$ \\
\hline Soft & 1.59 & 6.25 & 5.55 & 6.76 & - \\
\hline Total & $4.49^{*}$ & 88.64 & 96.25 & 96.47 & $\begin{array}{c}\mathrm{S}<\mathrm{C}, \mathrm{S}=\mathrm{L} \\
\text { and } \mathrm{L}=\mathrm{C}\end{array}$ \\
\hline Wt. of Sepioteuthis australis & 0.717 & 2.33 & 1.65 & 2.09 & - \\
\hline No. of Sepia spp. & $18.43^{* * *}$ & 12.9 & 17.29 & 26.01 & $\mathrm{~S}=\mathrm{L}<\mathrm{C}$ \\
\hline Wt. of Sepia spp. & $19.08^{* * *}$ & 2.077 & 3.27 & 5.16 & $\mathrm{~S}=\mathrm{L}<\mathrm{C}$ \\
\hline No. of Portunus armatus & $10.81^{* * *}$ & 467.67 & 516.43 & 781.83 & $\mathrm{~S}=\mathrm{L}<\mathrm{C}$ \\
\hline Wt. of Portunus armatus & $21.91^{* * *}$ & 32.82 & 31.14 & 62.46 & $\mathrm{~S}=\mathrm{L}<\mathrm{C}$ \\
\hline Wt. of mixed bycatch & 0.08 & 21.01 & 21.16 & 21.93 & - \\
\hline Wt. of total bycatch & $17.75^{* *}$ & 52.28 & 54.71 & 88.45 & $\mathrm{~S}=\mathrm{L}<\mathrm{C}$ \\
\hline
\end{tabular}




\section{DISCUSSION}

In an earlier paper (Broadhurst et al. 2007), we described a bycatch reduction framework that involves identifying and then testing the limits within which prospective BRDs should be constructed. Such limits encapsulate known parameters concerning the sizes and behaviours of target and bycatch species and operational factors within the fishery. The current study successfully identified these limits for bycatch issues concerning Sepia spp. and Portunus armatus in the Spencer Gulf prawn fishery.

The results firstly confirmed that, notwithstanding the issues concerning $S$. apama and $P$. armatus (which were caught here in quantities typical of those experienced in the fishery - see Currie et al. 2009), the Spencer Gulf fishery (with a prawn-tobycatch ratio of $\sim 1: 1.16$ by weight) is very selective compared to many global penaeid-trawl fisheries (Alverson et al. 1994). Nevertheless, as for other trawl fisheries, it is clear that mechanical-separating BRDs, such as the Nordmøre-grid, can reduce bycatch in this fishery, while maintaining penaeid catches. The large grid tested here certainly showed this - with $36.6,50.1$ and $38.1 \%$ reductions in the weights of Sepia spp., $P$. armatus and total bycatch, respectively, with no effect on the catches of Melicertus latisulcatus.

The small grid yielded predicted mean reductions in bycatches of Sepia spp., P. armatus and total bycatch that were greater than those of the large grid-although these differences were not statistically significant. However, the small grid also reduced catches of M. latisulcatus by $7.8 \%$. The small grid followed those designs previously tested and implemented in several fisheries (e.g. Isaksen et al. 1992, Broadhurst et al. 1996, Brewer et al. 1998, Silva et al. 2011), but we recognised that due to (1) the large quantities of $P$. armatus caught in Spencer Gulf, (2) their morphology and sharp exoskeletons and (3) their propensity to hold meshes with their chelipeds, the original design may have some limitations in the form of clogging caused by $P$. armatus and the associated loss of $M$. latisulcatus. By including the very long $(4 \mathrm{~m})$ guiding panel at a low seam angle $\left(\sim 26^{\circ}\right)$, we hypothesised that $M$. latisulcatus would fatigue and orientate towards the bottom (Watson 1989), while the large numbers of $P$. armatus would have sufficient distance to be separated out as they approached the grid.

The above hypothesis was realized in our study, although some $P$. armatus were entangled at the base of the small grid, perhaps due to the relatively steep transition in panel-to-grid angle. The results for the large, shallow-angled grid support this supposition because there was substantially less blockage in this $\mathrm{BRD}$. Increasing the length of the grid also provided considerably more sorting area, which perhaps facilitated the retention of $M$. latisulcatus to the same degree as the control.

Despite the success of the large grid, additional research is required to improve its performance, including perhaps slight modifications to the guiding panel to stop teleosts and $P$. armatus from swimming back up to its top edge and/or smooth canvas sides and thus further reduce $P$. armatus entanglement. Another worthwhile modification might be to reduce the bar spacing, at least until $M$. latisulcatus catches are affected, or even potentially beyond this point if other management tools can be implemented. For example, we worked within an objective of retaining $100 \%$ of M. latisulcatus, while maximising Sepia spp. exclusion. Simply reducing the bar spacing could exclude more Sepia spp. (and perhaps some teleosts) and, because any escaping $M$. latisulcatus should survive, their loss could be compensated by their subsequent capture during additional fishing nights outside the $S$. apama migration.

As part of any future work to test refined BRDs, escape mortalities need to be estimated for M. latisulcatus and especially for the soft-bodied Sepia spp., since the ultimate utility of BRDs requires large proportions of escaping individuals to not only survive, but also continue to successfully spawn. We also acknowledge that our study was done over a short period of time and at only a few locations in Spencer Gulf, and future work is currently being planned to examine the broader spatial and temporal applicability of refined BRDs.

Notwithstanding the need for ongoing research, the present study has shown that a modified Nordmøre-grid could go a long way towards reducing the bycatch of $S$. apama and $P$. armatus in this fishery, with minimal commercial impacts. Combined with the existing management tools used in the fishery (fixed and real-time spatial and temporal closures) and industry-developed technical solutions (the crab bag and on-board sorting systems), such a BRD should significantly contribute to the optimal resource management that is characteristic of this fishery.

Acknowledgements. This work was funded by the Australian Fisheries Research and Development Corporation (Project No. 2013/052). The authors acknowledge the skill and professionalism of the skipper (Clinton Scharfe) and crew (Max Sturman, Bob Lovett and David Verran) of the 'Grozdana B' for ensuring that the field work was successful. 
Geoff Johnson and Steve Everson are thanked for their skills in fabricating the BRDs. We also thank Simon Clark, Sean Sloan, Brad Milic, Craig Noell, Bronwyn Gillanders and Russell Miller for their assistance and helpful discussions, and Andy Revill and 2 anonymous referees for their helpful comments on the manuscript. Finally, we thank the New South Wales Department of Primary Industries for supporting M.B.

\section{LITERATURE CITED}

Alverson DL, Freeberg MH, Murawski SA, Pope JG (1994) A global assessment of fisheries by-catch and discards. FAO Fisheries Tech Pap 339, FAO, Rome

Barratt I, Allcock L (2012) Sepia apama. In: IUCN 2013. IUCN Red List of threatened species, Version 2013.2. Available at www.iucnredlist.org (accessed 17 May 2014)

Brewer D, Rawlinson N, Eayrs S, Burridge C (1998) An assessment of bycatch reduction devices in a tropical Australian prawn trawl fishery. Fish Res 36:195-215

Broadhurst MK (2000) Modifications to reduce bycatch in prawn trawls: a review and framework for development. Rev Fish Biol Fish 10:27-60

Broadhurst MK, Kennelly SJ, Isaksen B (1996) Assessments of modified codends that reduce the by-catch of fish in two estuarine prawn-trawl fisheries in New South Wales, Australia. Fish Res 27:89-111

Broadhurst MK, Kennelly SJ, O'Doherty G (1997) Specifications for the construction and installation of two by-catch reducing devices (BRDs) used in New South Wales prawn-trawl fisheries. Mar Freshw Res 48:485-489

Broadhurst MK, Kennelly SJ, Gray CA (2007) Strategies for improving the selectivity of fishing gears. In: Kennelly SJ (ed) By-catch reduction in the world's fisheries. SpringerVerlag, Dordrecht, p 1-18

Currie DR, Dixon CD, Roberts SD, Hooper GE, Sorokin SJ,

Editorial responsibility: Eric Gilman,

Honululu, Hawaii, USA
Ward TM (2009) Fishery-independent by-catch survey to inform risk assessment of the Spencer Gulf Prawn Trawl Fishery. SARDI Publication No. F2009/000369-1, SARDI Research Report Series No. 390, South Australian Research and Development Institute, West Beach, SA

Dixon C, Clark S, Hill W (2014) South Australian prawn fisheries improving environmental practices. Final report. Caring For Our Country 2012/13 Investment Project: OC13-00090, Spencer Gulf and West Coast Prawn Fisherman's Association, Port Lincoln, SA

Hall MA (1996) On bycatches. Rev Fish Biol Fish 6:319-352

Isaksen B, Valdemarsen JW, Larsen RB, Karlsen L (1992) Reduction of fish bycatch in shrimp trawl using a rigid separator grid in the aft belly. Fish Res 13:335-352

Kelleher K (2005) Discards in the world's marine fisheries. An update. FAO Fisheries Tech Pap No. 470, FAO, Rome

Kennelly SJ (2007) By-catch reduction in the world's fisheries. Springer-Verlag, Heidelberg

McShane P (1997) Transfer of by-catch reduction technology to South Australian prawn fisheries. Final FRDC Report No. 96/254.02, South Australian Research and Development Institute, West Beach, SA

Silva CNS, Broadhurst MK, Schwingel A, Dias JH, Cattani AP, Spach HL (2011) Refining a Nordmøre-grid for a Brazilian artisanal penaied-trawl fishery. Fish Res 109: 168-178

Steer MA, Gaylard S, Loo M (2013) Monitoring the relative abundance and biomass of South Australia's giant cuttlefish breeding population. FRDC Final Report 2011/054, SARDI Publication No. F2013/000074-1, SARDI Research Report Series No. 684, South Australian Research and Development Institute, West Beach, SA

Watson JW (1989) Fish behaviour and trawl design: potential for selective trawl development. In: Campbell CM (ed) Proceedings of the world symposium on fishing gear and fishing vessels. Marine Institute, St. Johns, p 25-29

Submitted: May 20, 2014; Accepted: July 26, 2014

Proofs received from author(s): October 25, 2014 